\title{
CHARMONIUM SPECTROSCOPY FROM LATTICE QCD
}

\author{
CARLETON DeTAR* \\ Department of Physics and Astronomy, University of Utah, Salt Lake City, UT 84112, USA
}

I give a short review of the current status of lattice QCD calculations of the spectrum of charmonium.

Keywords: charmonium; quarkonium; lattice QCD.

PACS numbers: 14.40.Lb, 14.40.Pq, 12.38.Gc

\section{Introduction}

In recent years the numerical simulation of lattice quantum chromodynamics (QCD) has developed into an enormously successful method for obtaining precise values of several Standard Model parameters. It is the only truly ab initio method available for doing nonperturbative studies of QCD. It provides a unified framework for treating mesons and baryons involving charm and bottom quarks, whether in a heavy-light or heavy-heavy configuration.

For charm physics the primary phenomenological objectives of lattice QCD are to help in the discovery and characterization of excited states, including exotics, to determine decay constants ${ }^{1,2}$ and form factors, and to calculate electromagnetic transition rates. Theoretical objectives include providing guidance for effective field theory.

Since the last Charm conference there have been no new comprehensive numerical lattice studies of exotics and excited quarkonium states. So I will review the current status only briefly. There has been exciting progress, however, in the high precision determination of masses of several of the lowest lying states involving heavy quarks. Progress here is, of course, prerequisite to an accurate determination and characterization of excited states.

Lattice simulations have their limitations. While static properties, including masses and matrix elements are relatively easy to calculate, scattering processes, including charmonium production, inclusive processes, and multihadronic (more than two-body) decays are very difficult.

In addition to the usual challenges of extrapolating to the continuum and physical light quark masses, heavy quark simulations face special difficulties. The lattice

*Temporary address: University of Tsukuba, Tsukuba, Japan 

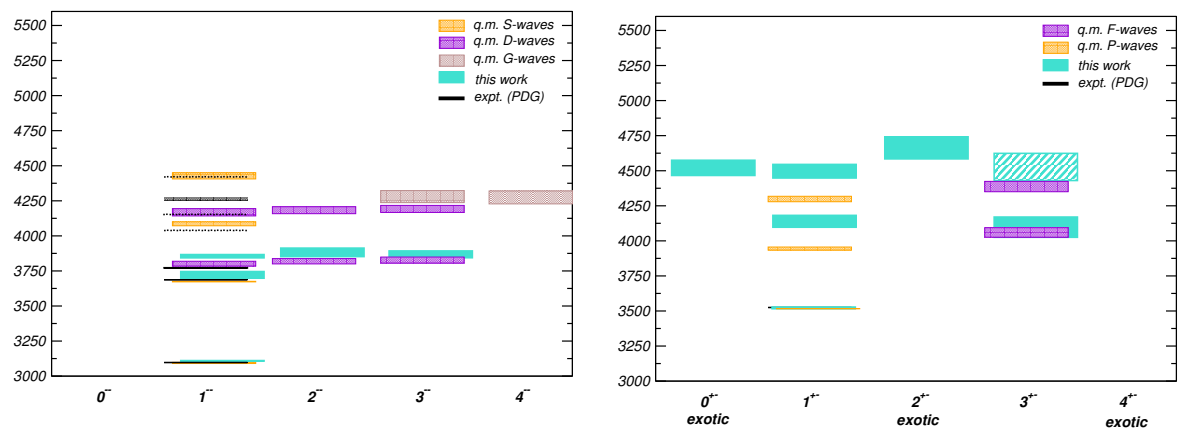

Fig. 1. Charmonium excitation spectrum in MeV from Ref. 7. Left: $J^{--}$states. Right $J^{+-}$states including exotics. Lattice results (blue) are compared with PDG values (black) and various quark model results (purple and orange). Color online.

spacing $a$ introduces a momentum cut off of order $1 / a$, typically less than $2-3 \mathrm{GeV}$ with today's lattices. As the quark mass $M$ approaches the cut off, discretization errors grow. Such mass-related errors can be controlled by a suitable formulation of the Dirac action for the heavy quark. Among them are (1) a lattice version of nonrelativistic $\mathrm{QCD}^{3}$, which converges rather slowly for charm, but well for bottom, (2) the Fermilab action ${ }^{4}$ and its improvements, ${ }^{5,6}$ good for both charm and bottom, and (3) the highly improved staggered quark action (HISQ) with discretization errors of order $\mathcal{O}\left(\alpha_{s}^{2}\left(a M_{c}\right)^{2}\right)$, good for charm, but not so good for bottom with today's lattices.

\section{Excitation spectrum and exotics}

In 2008 Dudek et al. ${ }^{7}$ published results of a comprehensive lattice QCD study of the excitation spectrum of charmonium. In order to access excited states they introduced a large set of interpolating operators and used a "variational" method to determine their masses.

How well does this work? Figure 1 (left) gives an impression. Consider the $1^{--}$ channel, where the ground state $J / \psi$ has a very clean signal. Six states were found, but only the first three shown were unambiguous enough that the authors were willing to associate them with known levels, namely the $J / \psi, \psi(2 S)$ and $\psi(3 S)$. The lattice levels were generally higher than experiment and the discrepancies grew from 12 to $82 \mathrm{MeV}$. They were also higher than quark model determinations, as shown. In the $P C=+-$ channel this study also produced a couple spin-exotic states as shown in Fig. 1. See Ref. 7 for more states.

These results are pioneering, but they are deficient for a number of reasons. Sea quark effects were omitted, the calculation was done at only one lattice spacing, so an extrapolation to the continuum is not possible, and open charm states were ignored. The Hadron Spectrum Collaboration is remedying these shortcomings. ${ }^{8}$

To include sea quarks and allow extrapolation to the continuum and to physical light quark masses requires generating gauge field configurations (ensembles) with 

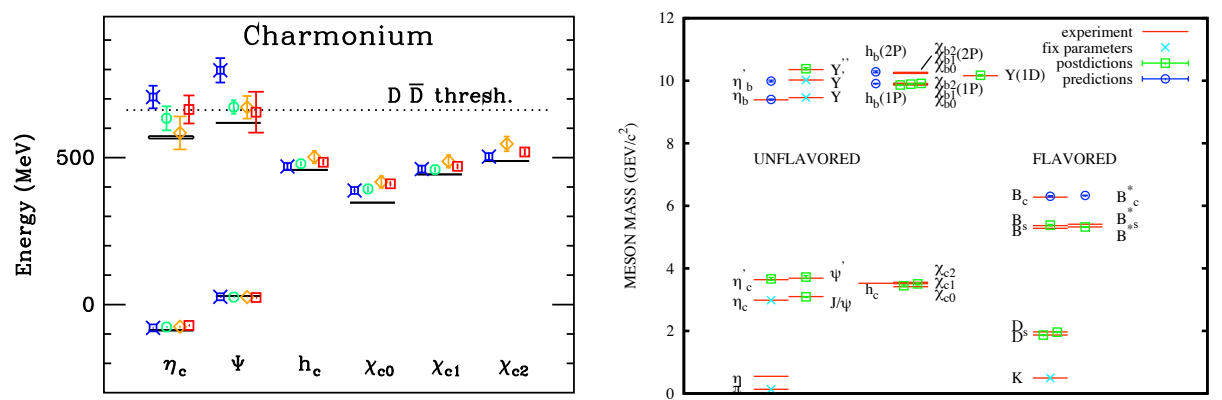

Fig. 2. Left: Charmonium levels from Ref. 10 based on splittings from the spin-averaged $1 S$ level with quark masses determined from the $D_{s}$ and light pseudoscalar mesons and scale determined from upsilon splittings. Results for four lattice spacings from $0.18 \mathrm{fm}$ (red) to $0.09 \mathrm{fm}$ (blue) are shown. Black lines are PDG values. Right: complete low-lying heavy and light meson spectrum from Ref. 11 showing five masses used to fix four quark masses and the lattice scale and three masses predicted in advance of experiment.

various lattice spacings and light quark mass combinations. The MILC collaboration makes publicly available a large archive of such ensembles with lattice spacing ranging from $0.15 \mathrm{fm}$ to $0.045 \mathrm{fm}$ and light quark mass ratios $m_{u d} / m_{s}$ ranging down to 0.05 , compared with the physical value of approximately $0.037 .{ }^{9}$

MILC gauge configurations are being used by the FNAL/MILC and HPQCD collaborations to study the quarkonium spectrum. The FNAL/MILC campaign currently uses Fermilab quarks for both charm and bottom and HPQCD uses HISQ charm quarks and NRQCD bottom quarks. Most of the FNAL/MILC results presented here are from last year's study, ${ }^{10}$ which, like the HPQCD study, used a limited set of interpolating operators and looked at only the low-lying states. A new FNAL/MILC campaign currently under way is aimed at excited as well as ground states using a large set of interpolating operators, higher statistics, smaller lattice spacings, and more accurate heavy quark mass tuning. I will give a preview of the improvement we hope to obtain.

\section{Low-lying levels}

Broadly speaking, lattice simulations do quite well reproducing the low-lying hadronic states. Figure 2 gives an overview of the charmonium spectrum of the FNAL/MILC collaboration (left) ${ }^{10}$ and the full gold-plated meson spectrum of the HPQCD collaboration ${ }^{11}$ (right). To appreciate the precision of the calculation, we turn to a finer mass scale in the remainder of this minireview.

Historically, the hyperfine splitting of the $1 S$ state has proven to be a stringent test of lattice methodology. In Fig. 3 recent results of the FNAL/MILC collaboration (left) ${ }^{10}$ are compared with still more recent results of the HPQCD collaboration (right) ${ }^{12}$. The FNAL/MILC calculation was based on Fermilab quarks. The extrapolated value is $117(11) \mathrm{MeV}$. In this result annihilation effects are not included. The HPQCD result is based on HISQ charm quarks. The pink band indicates the 

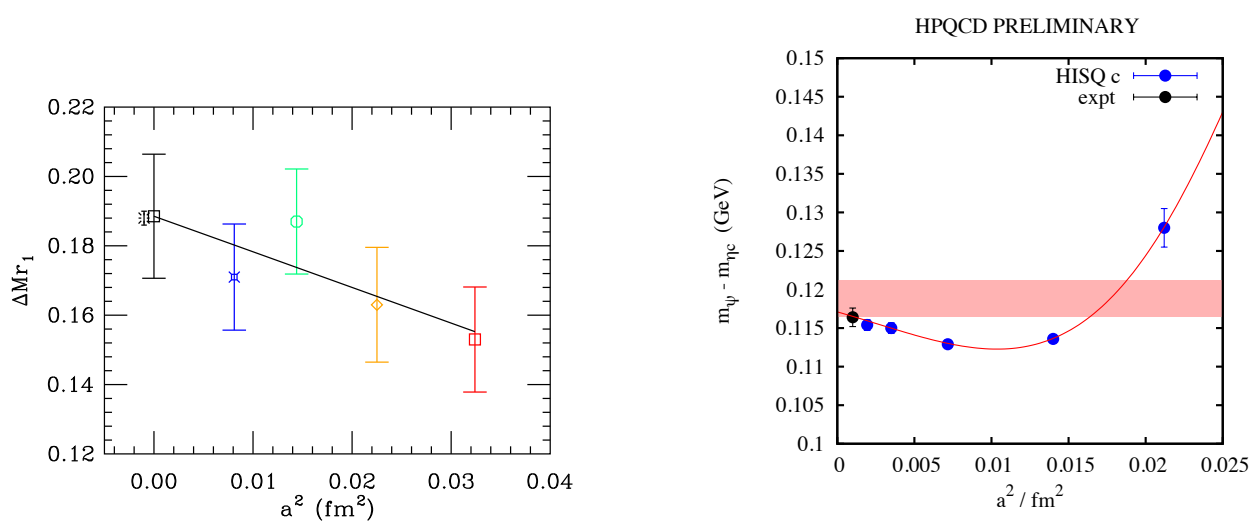

Fig. 3. Hyperfine splitting in charmonium extrapolated to zero lattice spacing. PDG values are in black. Left: FNAL/MILC ${ }^{10}$. Units are $r 1 \approx 1.58 \mathrm{Gev}^{-1}$. Right: $\mathrm{HPQCD}^{12}$.
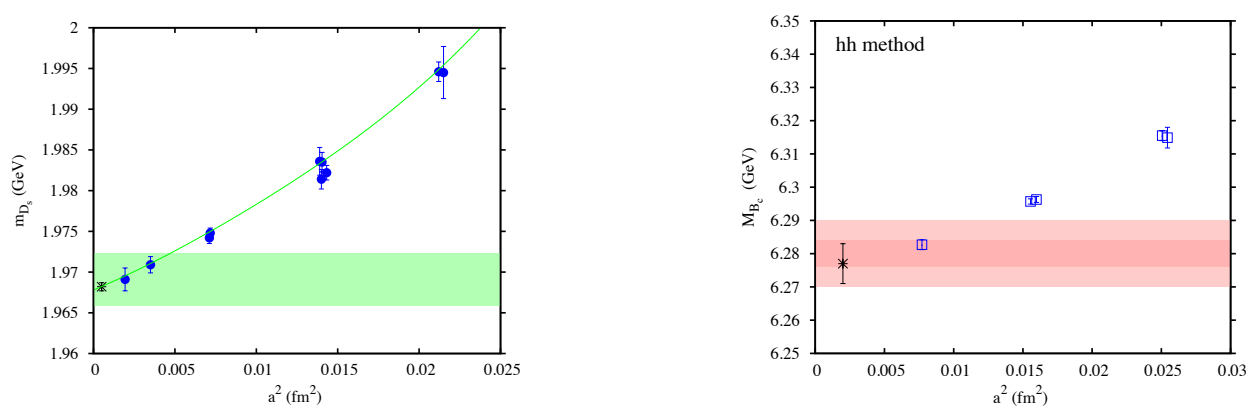

Fig. 4. Recent HPQCD results for the $D_{s}$ mass (left) ${ }^{14}$ and $B_{c}$ (right $)^{15}$.

full error budget $( \pm 2 \mathrm{MeV})$, and includes a small increase due to annihilation, as suggested by perturbation theory. Determining level shifts due to annihilation is difficult. A recent nonperturbative (lattice) result estimates, instead, a decrease of $1-4 \mathrm{MeV}$ from charm annihilation. ${ }^{13}$ Thus, apparently, we have reached the point where annihilation effects cannot be ignored.

Further examples of achievable precision in gold-plated quantities are shown in Fig. 4. The $D_{s}$ mass is obtained to $3 \mathrm{MeV}$ accuracy from the mass splitting $M(c \bar{s})-\frac{1}{2} M\left(\eta_{c}\right),{ }^{14}$ and the $B_{c}$ mass is obtained to $10 \mathrm{MeV}$ accuracy from the splitting $M\left(B_{c}\right)-M(b \bar{b}) / 2-M\left(\eta_{c}\right) / 2 .{ }^{15}$

Going beyond gold-plated quantities, the FNAL/MILC simulation results for the spin-averaged $2 S-1 S$ splitting, shown in Fig. 5 (left) disagrees with the experimental value. ${ }^{10}$ Could this be a result of complications from the nearby open charm threshold? Those simulations do not currently treat two-body scattering states. A recent calculation by Bali and Ehmann with a small basis set suggests that they may be important for even states well below threshold, but further study is needed. ${ }^{16}$ 

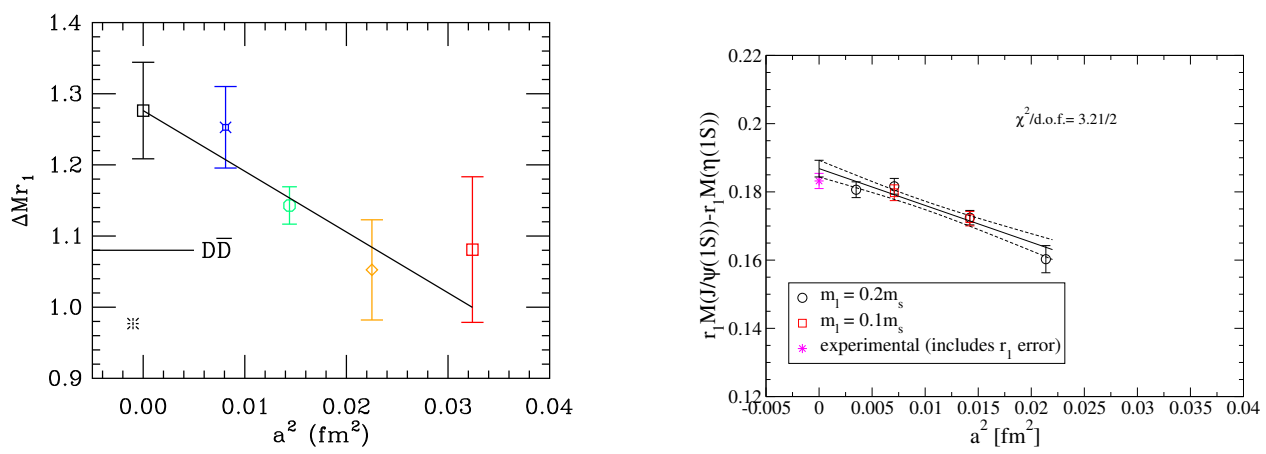

Fig. 5. Left: $\overline{\mathbf{2 S}}-\overline{\mathbf{1 S}}$ splitting from Ref. 10 showing possible complications from open charm. Right: Sample FNAL/MILC preliminary result for the $1 S$ hyperfine splitting from the new analysis with much reduced errors.

\section{Conclusions and Outlook}

We have seen dramatic improvement in our ability to reproduce the masses of the lowest lying charmonium states. This has been brought about by the use of improved quark actions, higher statistics, accurate tuning of the heavy quark masses, smaller lattice spacing, and the availability of gauge field ensembles that support an extrapolation to physical sea quark masses and zero lattice spacing. New analysis campaigns with multiple interpolating operators are underway both by the Hadron Spectrum Collaboration and the Fermilab Lattice/MILC collaborations. A preview of the hyperfine splitting from this new analysis is shown in Fig. 5 (right), a considerable improvement over Fig. 3 (left). These campaigns should provide more accurate information about excited and exotic states. Treating two-body scattering states will continue to be a long-term challenge.

\section{References}

1. J. Simone, these proceedings.

2. Hee Chang $\mathrm{Na}$, these proceedings.

3. G. P. Lepage et al., Phys. Rev. D 46, 4052 (1992) [arXiv:hep-lat/9205007].

4. A. X. El-Khadra, A. S. Kronfeld and P. B. Mackenzie, Phys. Rev. D 55, 3933 (1997) [arXiv:hep-lat/9604004].

5. M. B. Oktay and A. S. Kronfeld, Phys. Rev. D 78, 014504 (2008) [arXiv:0803.0523 [hep-lat]].

6. M. B. Oktay, PoS LAT2010 (to be published).

7. J. J. Dudek et al., Phys. Rev. D 77, 034501 (2008) [arXiv:0707.4162 [hep-lat]].

8. S. Ryan, PoS LAT2010 (to be published).

9. A. Bazavov et al., Rev. Mod. Phys. 82, 1349 (2010) [arXiv:0903.3598 [hep-lat]].

10. T. Burch et al., Phys. Rev. D 81, 034508 (2010) [arXiv:0912.2701 [hep-lat]].

11. E. B. Gregory et al., Phys. Rev. Lett. 104, 022001 (2010) [arXiv:0909.4462 [hep-lat]].

12. E. Follana (HPQCD Collaboration) PoS LAT2010 (to be published).

13. L. Levkova and C. DeTar, arXiv:1012.1837 [hep-lat].

14. C. T. H. Davies et al., arXiv:1008.4018 [hep-lat].

15. E. B. Gregory et al., arXiv:1010.3848 [hep-lat].

16. G. Bali and C. Ehmann, arXiv:0911.1238 [hep-lat]. 